\title{
Performance of Energy Detection and Matched Filter Receivers in Human Body Communication Channel
}

\author{
Mohamad Abou El-Nasr, Heba Shaban and Mohammed Al-Abyadh \\ Department of Computer Engineering, Electronics and Communications Engineering, \\ Arab Academy for Science, Technology and Maritime Transport (AASTMT), Alexandria, Egypt
}

Received 2012-11-11, Revised 2012-12-20; Accepted 2012-12-20

\begin{abstract}
This study studies the Bit-Error-Rate (BER) performance of matched filter and energy detection receivers in Human Body Communication (HBC) channel model. HBC, as defined by the IEEE 802.15 Task Group (TG) 6, is a Body Area Network (BAN) at frequency band 5-50 MHz, where communication is from body surface to body surface. The Bit-Error-Rate (BER) performance is investigated for Energy Detection (ED) and Matched Filter (MF) receivers. BER performance is studied and compared for Binary Pulse-Position Modulation (BPPM) and Binary Phase Shift Keying (BPSK). Moreover, the effect of varies channel parameters on the BER of studied receivers is investigated, including ground plate area at Transmit (Tx) and Receive (Rx) antennas and distance between Tx and receive antennas through air and body. Results show that the BER of both receivers is not affected by varying channel parameters.
\end{abstract}

Keywords: Body Area Network (BAN), Bit Error Rate (BER), Human Body Communication (HBC) Channel

\section{INTRODUCTION}

Recently, Body Area Networks (BANs) have received significant attention in both medical and consumer applications. The IEEE has set up a working group 802.15.6 for standardization of the wireless BAN in the end of 2007. The IEEE 802.15.6 task group is defining the physical and data link layer for BANs. The task group identified four distinct communication scenarios between devices, namely implant to implant, implant to on/off body, on-body to on-body and on-body to off body (Yazdan-Doost and Pour, 2009). BAN communication is of importance to medical and healthcare services, where various medical and health-care data is transmitted on the human body from vital sign sensors with high security and reliability. Channel models of the IEEE 802.15.6 have been published in 2009 (IEEE, 1980). Sensing and actuating devices are becoming sufficiently small to allow multiple sensors to be attached to the body. A network of such sensors around the body is referred to as a wireless BAN. It is expected that the main applications of BANs will be in biomedical and sporting domains, as discussed in the IEEE 802.15.6 working group (Zhen et al., 2009). The close proximity of the transceivers to the human body necessitates a lowpower demand to any BAN and requires the wireless channel be fully defined (Hall and Hao, 2006).

The BAN channels of the IEEE 802.15.6a task group are intended to develop BAN for medical and nonmedical devices that could be placed inside or on the surface of human body using different frequency bands for implant to implant, implant to surface, implant to external and body surface to body surface (Line-OfSight (LOS) and Non-Line-Of-Sight (NLOS). The channel model for BAN is required to evaluate the performance of different physical layer proposals and important for the development of any communication link (Yazdan-Doost and Pour, 2009). The physical Corresponding Author: Mohamad Abou El-Nasr, Department of Computer Engineering, Electronics and Communications Engineering, Arab Academy for Science, Technology and Maritime Transport (AASTMT), Alexandria, Egypt 
layer as proposed by the IEEE 802.15.6 comes in three different forms. The first is Narrowband Physical (NBPHY), which oc-cupies the 402-405 $\mathrm{MHz}$ range and primarily corresponds with communication with any devices that are implanted into the body. These devices can be nodes directly under the skin or deep within the body (Yazdan-Doost and Pour, 2009; IEEE, 1980). The second band is Ultra Wideband (UWB), which operates in the 3.1-10.6 GHz frequency band. UWB is used primarily to communicate between the body surfaces to any external device but also can be used to communicate to nodes within the body. The third category is Human Body Communications (HBC). HBC operates at the 13.5, 400, 600 and $900 \mathrm{MHz}$ frequencies as well as the 5-50 $\mathrm{MHz}$ frequency band (Yazdan-Doost and Pour, 2009; IEEE, 1980). HBC mode is primarily used to communicate between nodes on the body surface but also can be used to communicate to external devices.

Extensive work exists in the literature on the pathloss and power-delay of the both narrow-band and UWB wireless BANs at Industrial, Scientific and Medical (ISM) frequencies (Scanlon and Evans, 2001). However, to the best of the authors' knowledge, little to no literature is available for the performance evaluation of communication systems in the HBC channel. In this study we study the BER performance of energy detection and matched filter receivers in HBC body area network channel model of the IEEE 802.15.6a at frequency band 5-50 MHz.

\section{CHANNEL MODEL}

The channel impulse response of the human body communication is a suitable channel model to evaluate the BER performance of $\mathrm{ED}$ and MF receivers for different channel parameters for BAN applications. In the $\mathrm{HBC}$, a data signal is wireless transmitted through the body. In order to transfer a signal between Transmitter (Tx) and body or Receiver (Rx) and body, Tx and Rx have a metal plate signal electrode attached to the body. The signal electrode, attached to the body, transfers a signal from Tx to the body while transmitting signal, or from the body to $\mathrm{Rx}$ while receiving signal (IEEE, 1980). The HBC channel model is composed of the frequency response and the noise characteristics as shown in Fig. 1 (Baweja et al., 2011).

The frequency response is taken in the frequency range of 0-55 $\mathrm{MHz}$. The human body communication HBC channel impulse response is represented by (Baweja et al., 2011) equation 1:
$h(t)=h_{R} \cdot C_{h}$

where, $h_{R}$ is the reference impulse response and $C_{h}$ is a coefficient related to sizes of ground planes and distances between transmitter $\mathrm{Tx}$ and $\mathrm{Rx}$. The reference impulse response is given by (Baweja et al., 2011) equation 2:

$h_{R}(t)=A_{v} \cdot A \cdot \exp \left(-\left(t-t_{r}\right) / t_{0}\right) \cdot \sin \left(\pi \cdot\left(t-t_{r}-x_{c}\right) / w\right)$

where is a coefficient that represents fluctuation of the signal loss and its uniformly distributed $A_{v} \sim N\left(1,0.1 .6^{2}\right)$. $\mathrm{A}, \mathrm{t}, \mathrm{t}_{0}, \mathrm{X}_{\mathrm{c}}, \mathrm{w}$ and $\mathrm{t}_{\mathrm{r}}$ are constant values (Baweja et al., 2011). $C_{h}$ is given by equation 3 :

$$
\begin{aligned}
& \mathrm{C}_{\mathrm{h}}=\left(0.0422 . \mathrm{G}_{\mathrm{T}}-0.184\right) \cdot\left(0.0078 . \mathrm{G}_{\mathrm{R}}+0.782\right) \\
& {\left[\frac{120.49}{\mathrm{~d}_{\text {body } \left.+\mathrm{d}_{\text {body }} \text { (dair } / \mathrm{d}_{\text {body }}\right)}}\right]}
\end{aligned}
$$

The HBC channel model consists of a channel filter and a channel noise, where channel filter represents the signal loss by the human body. The HBC channel characteristics are represented by an impulse response to simultaneously model changes of transmitting signal's amplitude and phase by the channel filter. Frequency components of the impulse response are valid only between $0 \mathrm{MHz}$ and $50 \mathrm{MHz}$, so an input signal should be filtered with a low-pass filter to remove the frequency components out of the range. Also, when sampling the impulse response, a sampling rate should be over 250 $\mathrm{MHz}$ to accurately model the impulse response. The electromagnetic waves generated from various electronic devices cause a noise inside body due to an antenna effect of the human body, so the noise is added to a transmitting signal. In the HBC channel model, a power spectral density is used to represent level of the noise. A sample realization of HBC channel is shown in Fig. 2.

\section{SYSTEM MODEL}

The BER performance of MF and ED receivers is evaluated via simulations using MATLAB assuming Binary Pulse Position Modulation (BPPM) and Binary Phase Shift Key (BPSK) modulation schemes. The system model consists of one transmitter, channel of IEEE 802.15.6 and two types of receivers. The transmit-ted bits are in the form of $(1,0)$, are convolved with the channel of the human body communication and the performance of the two receiver types is evaluated. The received signal at the receiver input is given by equation 4 :

$$
y(t)=s(t) * h(t)+n(t)
$$


Mohamad Abou El-Nasr et al. / American Journal of Applied Sciences, 9 (12) (2012) 2021-2027

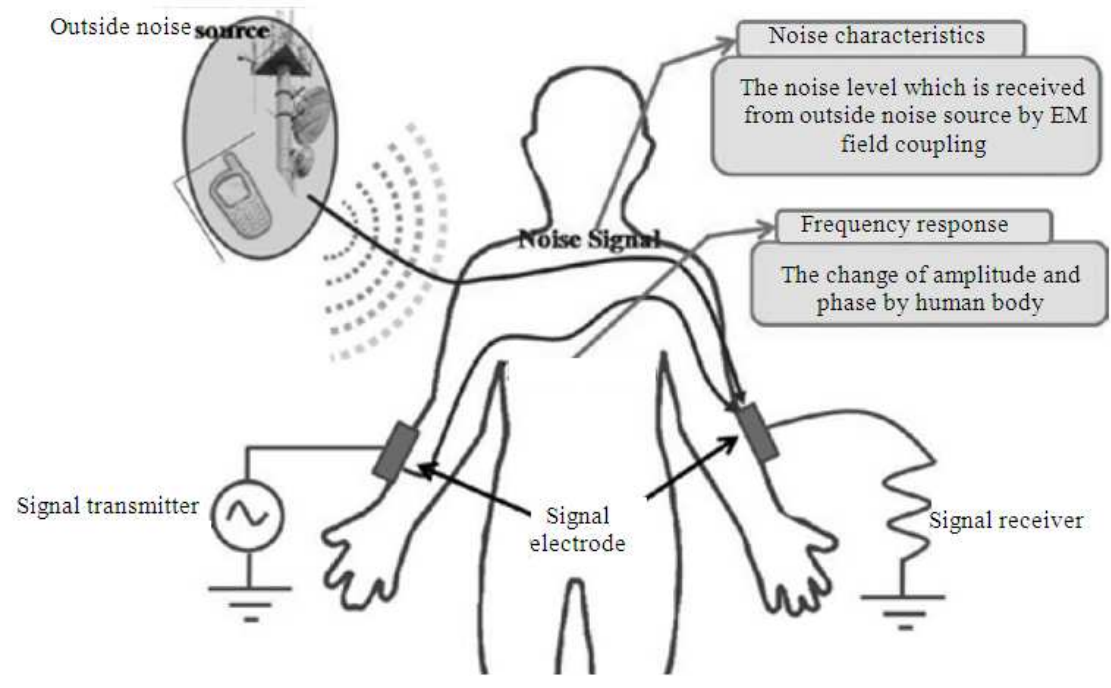

Fig. 1. Channel model for HBC (Baweja et al., 2011)

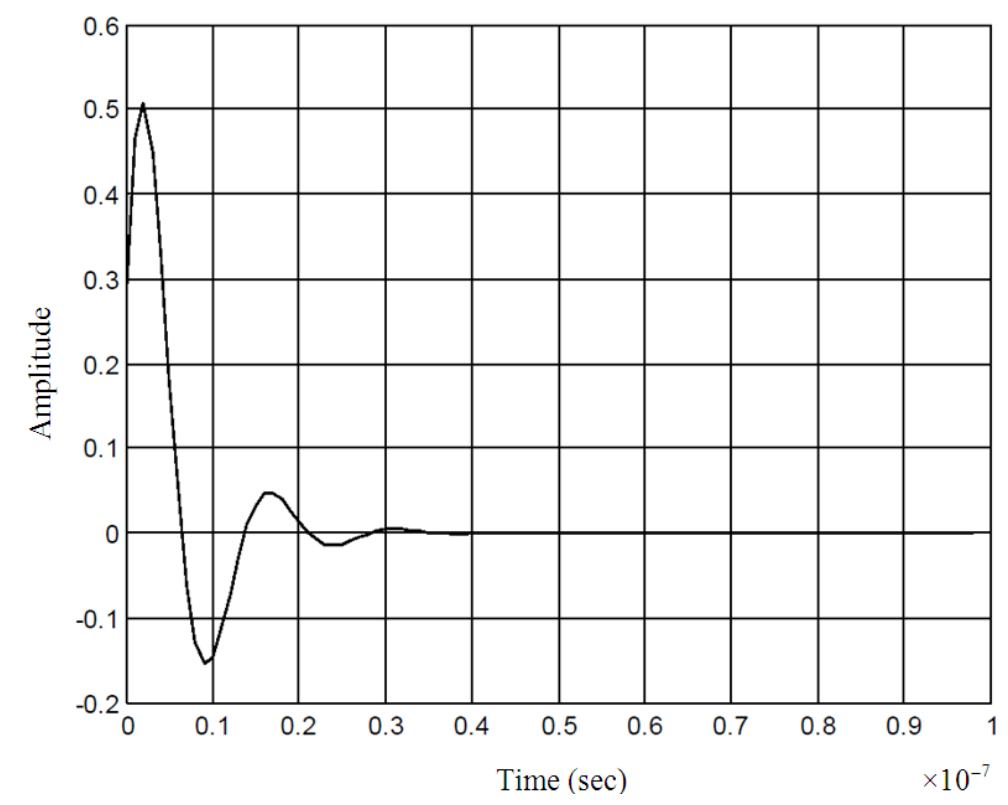

Fig. 2. Sample HBC channel realization

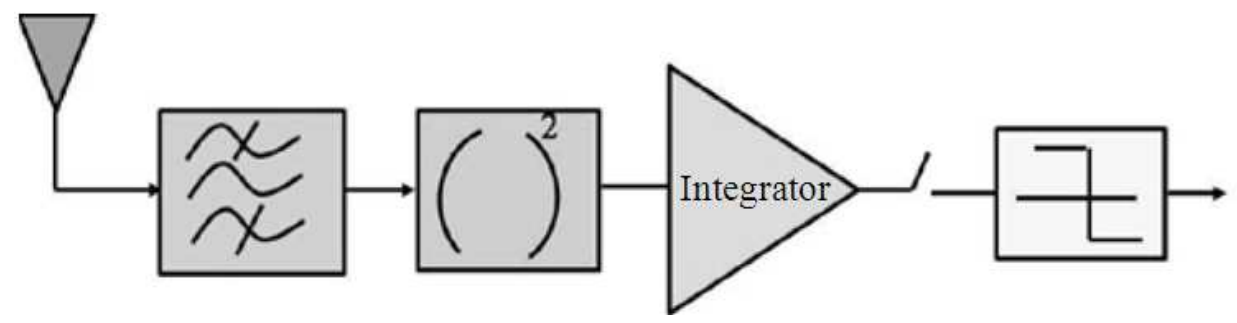

Fig. 3. Block diagram of energy detection receiver 


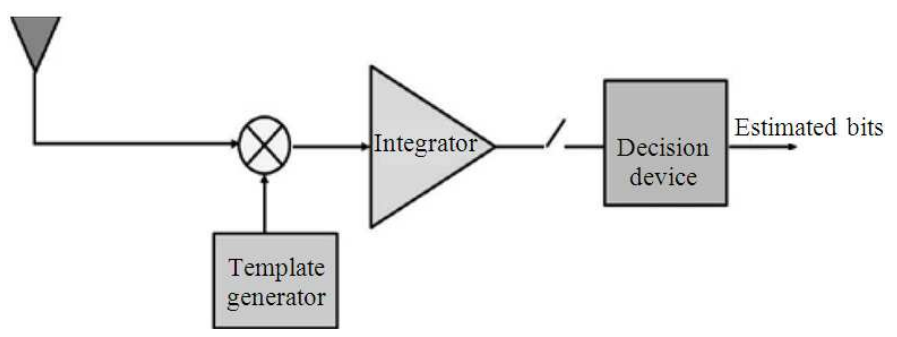

Fig. 4. Block diagram of matched filter receiver

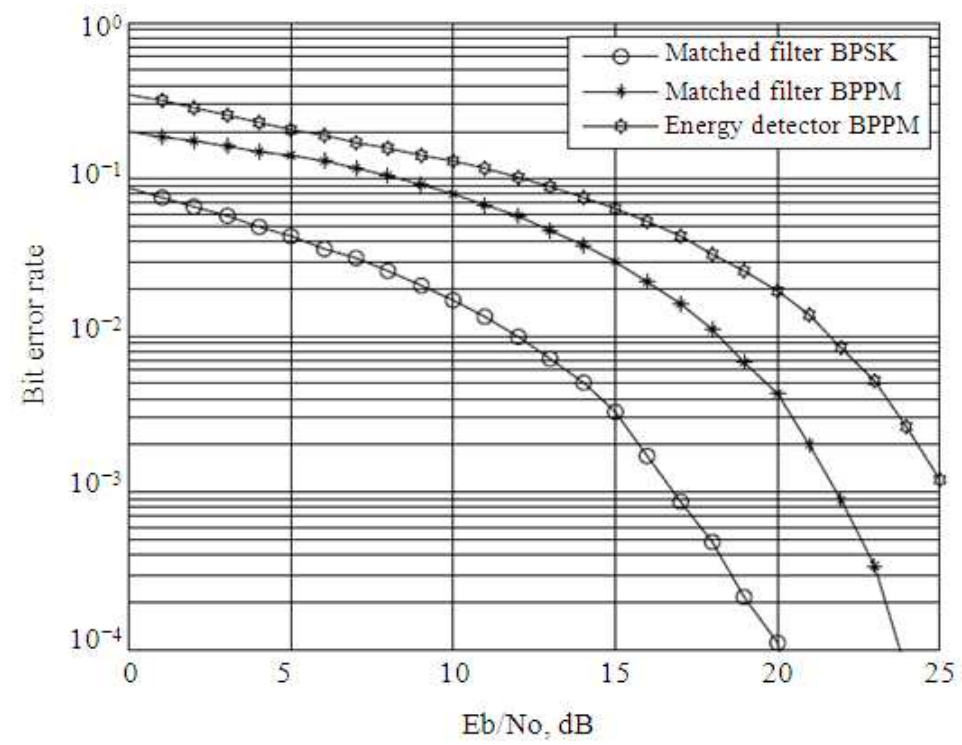

Fig. 5. BER performance comparison of $\mathrm{MF}$ and $\mathrm{ED}$ receivers in the $\mathrm{HBC}$ cha,nel model

Where, is the transmitted signal, $h(t)$ is the channel impulse response and $\mathrm{n}(\mathrm{t})$ is $\mathrm{AWGN}$. As was mentioned the HBC channel model consists of a channel filter and a channel noise. In the ED receiver the received signal is first passed through a band-pass filter for noise reduction and the integration is optimized for the channels. ED detects if the signal is present or not and it is a non-coherent detection, where low complexity receivers can be achieved at the expense of some performance degradation and are capable of collecting the energy from all the multipath components. Whereas, in the MF receiver, the received signal is convolved with the channel and the output is extracted at time $\mathrm{t}=\mathrm{T}$ and it is considered the optimum detector in Additive-White-Gaussian Noise (AWGN). In the presence of multipath fading, the use of receive and/or transmit diversity is normally preferred, however this is not the case for on-body communications since power consumption is an important factor.

BPPM is one of the most popular modulation schemes that have been considered for energy detectors. BPPM based implementation of energy detectors is achieved by passing the signal through a square-law device followed by an integrator and a decision mechanism. The input band-pass filter removes the outof band noise by selecting the center frequency $f_{c}$ and the bandwidth of interest. This is followed by a squaring device to measure the received energy and an integrator which determines the observation interval. A schematic block diagram of ED receiver is shown in Fig. 3. On the other hand, MF receiver uses convolutions between the received signal and a pre-stored template; the pulse and sampling at time $\mathrm{t}=\mathrm{T}$. The MF is the optimal AWGN channel, where it maximizes the Signal-to-Noise-Ratio (SNR) at the output. The block diagram of MF receivers is shown in Fig. 4. 


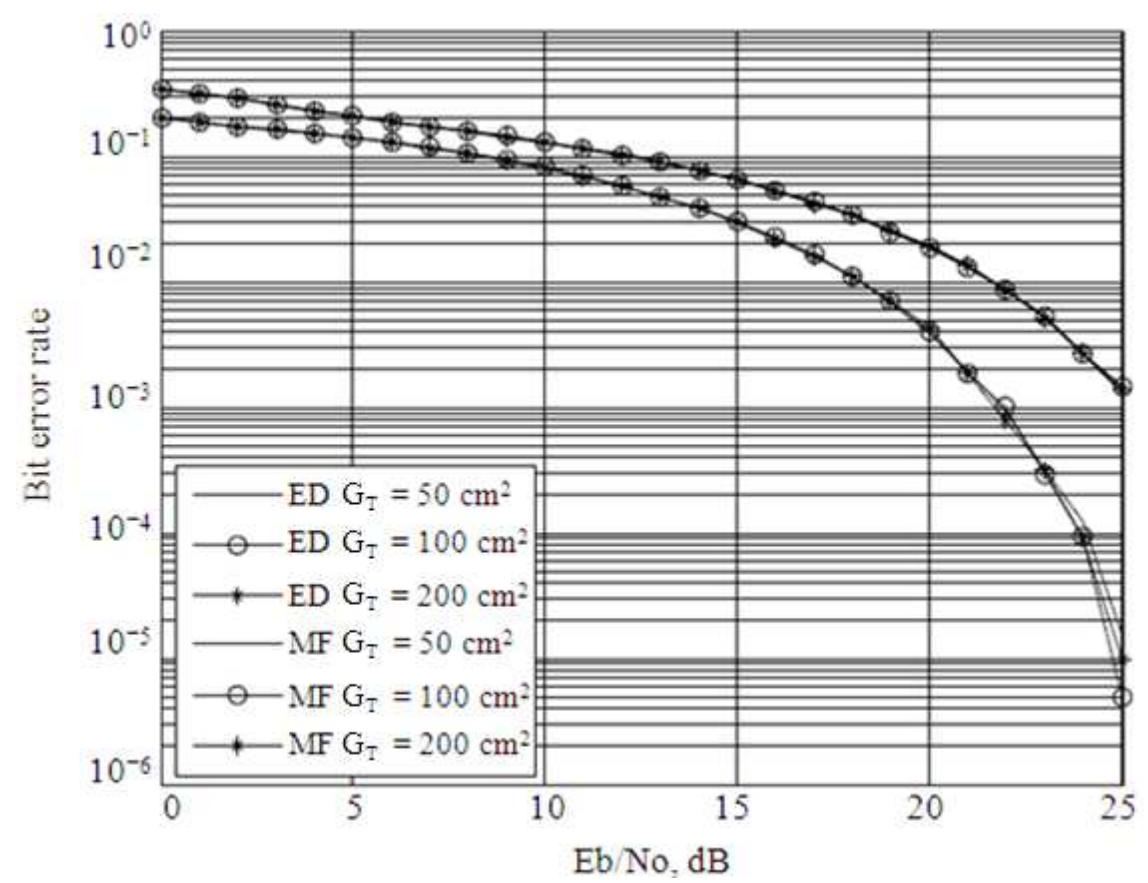

Fig. 6. BER performance comparison of $M F$ and $E D$ receivers in the $\mathrm{HBC}$ channel model for different values of ground plate areas at $\mathrm{Tx}_{\mathrm{T}} \mathrm{G}_{\mathrm{T}}$

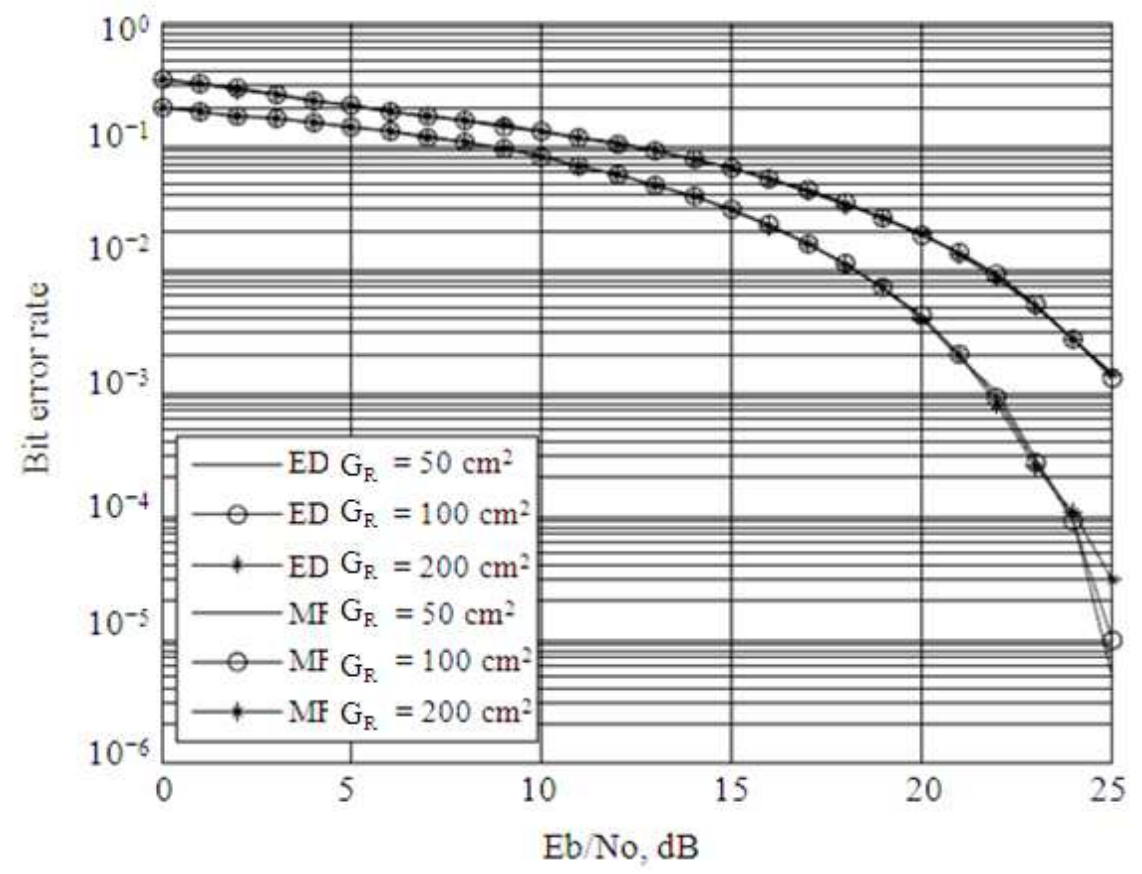

Fig. 7. BER performance comparison of $M F$ and $E D$ receivers in the $H B C$ channel model for different values of ground plate areas at $R x G_{R}$ In this study we evaluate the BER performance of the Communication (HBC) channel model parameters. $M F$ and ED receivers under different Human Body Numerical results are given. 


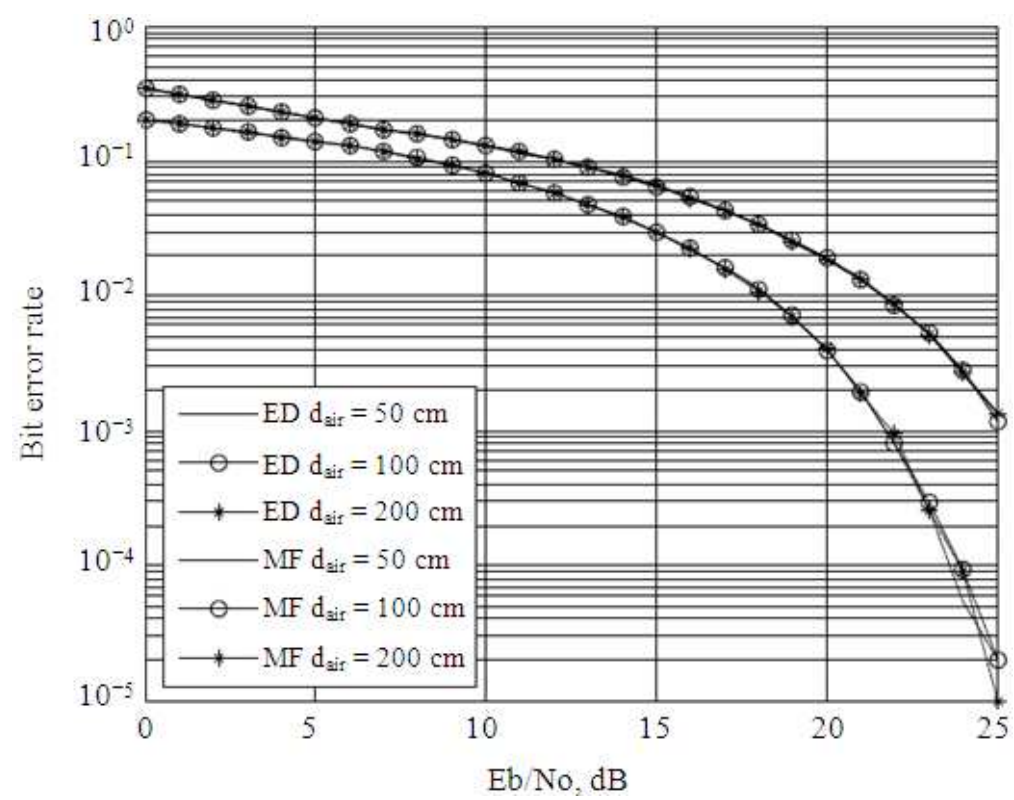

Fig. 8. BER performance comparison of MF and ED receivers in the HBC channel model for different values of Tx-Rx distance through air $d_{\text {air }}$

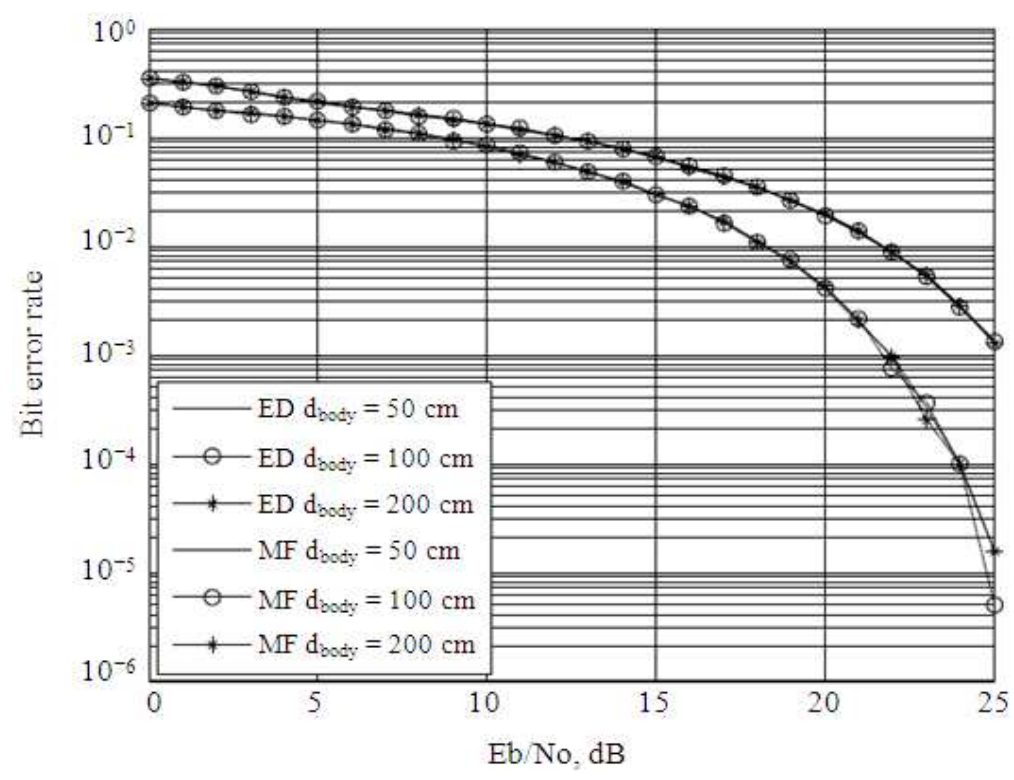

Fig. 9. BER performance comparison of MF and ED receivers in the HBC channel model for different values of Tx-Rx distance through body $\mathrm{d}_{\text {body }}$

\section{NUMERICAL RESULTS}

Provides numerical results obtained via Monte Carlo simulations using MATLAB for ED and MF receivers in the HBC channel defined by the IEEE 802.15.6a. First, the BER performance of $\mathrm{ED}$ and $\mathrm{MF}$ receivers is evaluated and compared assuming the default channel parameters for BPPM and BPSK modulation schemes, as shown in Fig. 5. Then, the channel parameters, namely ground plates for $\mathrm{Tx}$ and $\mathrm{Rx}$ and distance between $\mathrm{Tx}$ 
and Rx through body and air are varied and the BER performance is evaluated and compared. According to Fig. 5, MF receiver with BPPM outperforms ED receiver with the same modulation scheme with $3 \mathrm{~dB}$ at $\mathrm{BER}=2 \mathrm{e}-3$. In addition, $\mathrm{MF}$ receiver with BPSK provides a $5.5 \mathrm{~dB}$ SNR enhancement over the same receiver with BPPM scheme for the same BER. Figure 6-9 compare the BER of ED and MF receivers for different values of $G_{T}, G_{R}, d_{a i r}$ and $d_{\text {body }}$, respectively. As can be noted from figures, the BER performance of $\mathrm{ED}$ and MF receivers is insensitive to aforementioned parameters of the HBC channel.

\section{CONCLUSION}

This study studied and compared the BER performance of the MF and $\mathrm{ED}$ receivers in the $\mathrm{HBC}$ channel model for BANs. The BER performance of the studied receivers was evaluated for different channel parameters, namely the area of the ground plates at $T x$ and $R x$ and distance between $T x$ and $R x$ through body and air. The BER performance of the studied receivers was shown to be insensitive to channel parameter variations.

\section{REFERENCES}

Baweja, R., A. Sharma and P. Shukla, 2011. Simulation of constant modulus algorithm equalizer for human body communication channel. IJCA J. Elect., Inform. Commun. Eng., 6: 21-24.

Hall, P.S. and Y. Hao, 2006. Antennas and Propagation for Body-Centric Wireless Communications. 1st Edn., Artech House, London, ISBN-10: 1580534937, pp: 291.

IEEE, 1980. IEEE 802.15 WPANTM Task Group 6 (TG6) Body Area Networks. The Institute of Electrical and Electronics Engineers, Inc.

Scanlon, W.G. and N.E. Evans, 2001. Numerical analysis of bodyworn UHF antenna systems. IEEE Elect. Commun. Eng., 13: 53-64. DOI: 10.1049/ecej:20010203

Yazdan-Doost, K.Y. and K.S. Pour, 2009. Channel model for Body Area Network (BAN). New Generation Wireless Communication Research Center.

Zhen, B., M. Patel, S. Lee and E. Won, 2009. Body Area Network (BAN). 